\title{
Efficient adaptive integration of functions with sharp gradients and cusps in $n$-dimensional parallelepipeds
}

\author{
S. E. Mousavi ${ }^{1}$ and J. E. Pask ${ }^{2}$ and N. Sukumar ${ }^{1, *}$ \\ ${ }^{1}$ Department of Civil and Environmental Engineering, University of California, Davis, CA 95616, USA \\ ${ }^{2}$ Condensed Matter and Materials Division, Lawrence Livermore National Laboratory, Livermore, CA \\ 94550
}

SUMMARY

In this paper, we study the efficient numerical integration of functions with sharp gradients and cusps. An adaptive integration algorithm is presented that systematically improves the accuracy of the integration of a set of functions. The algorithm is based on a divide and conquer strategy and is independent of the location of the sharp gradient or cusp. The error analysis reveals that for a $C^{0}$ function (derivative-discontinuity at a point), a rate of convergence of $n+1$ is obtained in $\mathbb{R}^{n}$. Two applications of the adaptive integration scheme are studied. First, we use the adaptive quadratures for the integration of the regularized Heaviside function - a strongly localized function that is used for modeling sharp gradients. Then, the adaptive quadratures are employed in the enriched finite element solution of the all-electron Coulomb problem in crystalline diamond. The source term and enrichment functions of this problem have sharp gradients and cusps at the nuclei. We show that the optimal rate of convergence is obtained with only a marginal increase in the number of integration points with

\footnotetext{
* Correspondence to: N. Sukumar, Department of Civil and Environmental Engineering, University of California, One Shields Avenue, Davis, CA 95616. E-mail: nsukumar@ucdavis.edu
} 
respect to the pure finite element solution with the same number of elements. The adaptive integration scheme is simple, robust, and directly applicable to any generalized finite element method employing enrichments with sharp local variations or cusps in $n$-dimensional parallelepiped elements. Copyright (C) 2011 John Wiley \& Sons, Ltd.

KEY WORDS: adaptive quadrature; octree refinement; sharp gradients; cusps; partition-of-unity enrichment; density functional theory

\section{INTRODUCTION}

Functions with sharp gradients appear in the solution of problems with localization and cohesive process zones [1-3], shear bands $[4,5]$, thermal gradients [6-8], convection- and advection-diffusion problems [9-11], and in electronic structure calculations and ab initio materials modeling [12-14]. In the partition-of-unity [15] solution of these problems, such sharp gradients and cusps are efficiently resolved by incorporating enrichment functions that resemble the solution locally. As a result, efficient numerical integration of the basis functions and their gradients to form the system matrices becomes computationally demanding since one has to deal with strongly localized functions, instead of polynomial integrands. In many applications, the enrichment functions are the solution of local problems and known only numerically. Evaluation of such integrands can be extremely time-consuming, which points to the need for an efficient integration scheme. In this paper, we present an adaptive scheme for the integration of functions with sharp gradients and cusps. Adaptive integration algorithms accumulate integration points in regions with higher errors, and use fewer points where the integrand is smooth. Also, we are interested in domains that can be prescribed as a collection of hyperparallelepipeds, for example, parallelograms and parallelepipeds in two and three 
dimensions, respectively.

Adaptive integration schemes are normally recursive in nature and have a few common ingredients [16]: a quadrature rule that can be applied to the integration domain to provide a local estimate of the integration; a procedure to estimate the local integration error; a strategy to partition the integration domain into smaller divisions of the same shape; and a stopping criterion. The algorithm of Gander and Gautschi [17] over the interval and that of Berntsen et al. $[16,18]$ for a collection of triangles and tetrahedra are examples. Genz and Cools [19] proposed an algorithm for a vector-valued function over a combination of $n$-dimensional simplices. At each step, a subset of the simplices with the highest errors are selected and subdivided, and quadratures over the subdivisions are used to update the integral. The local error in Reference [19] is estimated by applying null quadrature rules - quadratures that (incorrectly) integrate to zero all polynomials up to degree $d$, and fail to do so for at least one polynomial of a higher degree $d+1[20,21]$. Herein, we use the difference in the integration of a function with two different tensor-product quadrature rules as the local error estimate, and proceed to subdivide a cell until the absolute error of integration of all the functions over each individual cell falls below a prescribed tolerance.

In this paper, we consider two types of local features. First, we focus on the regularized Heaviside function: this function has a sharp gradient, and by shrinking the size of the process zone, becomes strongly localized. Tornberg [22] and Patzák and Jirásek [1] proposed regularized forms of the Heaviside function that smear the strong discontinuity over a short distance on which the discontinuous function is approximated by a localized function. Oh et al. [23] introduced several smooth-piecewise-polynomial regularized discontinuous functions that can be used in the extended finite element method. Benvenuti et al. [2] presented a Copyright (C) 2011 John Wiley \& Sons, Ltd. Int. J. Numer. Meth. Engng 2011; 00:1-28 
method to integrate the regularized Heaviside function by the integration of the equivalent smooth functions. This is an extension of the method of Ventura [24] for the integration of discontinuous functions, which is however restricted to elements with constant Jacobian of the transformation. A more general treatment for the integration of arbitrary classes of functions, which is also adopted in this work, is adaptive integration using a posteriori error estimates: the integration points are concentrated close to the region where sharp gradients appear, and fewer points are used elsewhere [2].

Next, we consider functions with cusps. We pick the Coulomb problem in crystalline diamond and apply an enriched finite element (EFE) approach: Poisson's equation is solved with the electronic charge density as the source term, and the isolated atom solutions as the enrichment functions [14]. These functions have sharp gradients in the region close to the atomic sites, and have cusps at the nuclei. In one dimension, Möbius transformation has been used for the integration of functions with a peak at or near a boundary [25-27]. When Möbius transformation is applied to a standard quadrature over the interval, integration points are attracted toward the sharp gradient and more accurate results are obtained. However, this technique cannot be applied in higher dimensions. A well-known remedy in such cases is adaptive integration. Our adaptive integration scheme recursively performs a uniform refinement of the parent cell until the prescribed error tolerance over each subcell is met. The number of integration points in the adaptive quadratures is only marginally more than that obtained for the finite element solution with the same number of elements (and much lower accuracy), which points to the fact that numerical integration is not a bottleneck for the EFE solution of the problem.

The structure of this paper follows. In Section 2, the adaptive integration scheme is Copyright (C) 2011 John Wiley \& Sons, Ltd. Int. J. Numer. Meth. Engng 2011; 00:1-28 
introduced, followed by an error analysis in Section 3. It is shown that the algorithm is efficient for integrands with a cusp, such as $C^{0}$ functions of the form $f(\mathbf{x})=1-r$ or $f(\mathbf{x})=\exp (-\alpha r)$. The adaptive integration algorithm is used for the integration of the regularized Heaviside function in Section 4.1. In Section 4.2, an enriched finite element approach is applied to solve the all-electron Coulomb problem in crystalline diamond (charge density has a cusp at the nuclei). We close with a few concluding remarks in Section 5.

\section{ADAPTIVE INTEGRATION SCHEME}

Our quadrature construction algorithm is customized to meet the integration demands of high accuracy over parallelepipeds. A tensor-product quadrature based on a one-dimensional Gauss rule with five points in each direction is used to evaluate the local integrals. A tensor-product quadrature with eight points in each direction is used to compute the reference integral, and provides an estimate of the local integration error. The reason for choosing eight points in each direction is that a quadrature with six points might not be accurate enough to detect the integration error (due to the sharp gradients of the integrands); and a seven-point quadrature may suffer from the same odd-even defect of the quadratures as does a five-point quadrature. If the absolute error of integration is greater than the prescribed tolerance, the integration domain is uniformly divided into eight cells (in three dimensions) and the adaptive integration is performed over each cell recursively. This process is started with all the functions that need to be integrated, and at each step only those functions whose integration error is greater than the tolerance are passed to the next level, until all functions are integrated to the specified tolerance. Using the relative error as the stopping criterion may cause numerical difficulties in parts of the domain where the integral of at least one of the functions is close to zero. 
This problem was also reported in Reference [17], and was resolved by additionally checking the local estimate against the global estimate of the integration: if the contribution of the partition was small enough, further subdivision was circumvented. Such a problem is avoided by using the absolute error as the measure of accuracy. Note that the error tolerance in our quadrature construction scheme is only a stopping criterion, and may not represent the exact integration error. However, it provides a systematic means to improve the accuracy of the numerical integration until stable solutions are obtained. In a specific application, the appropriate tolerance depends on the overall accuracy required and the mesh resolution: as the mesh is refined, the tolerance (error per element) should be decreased proportionally to attain the same overall accuracy. Furthermore, if material parameters that appear in the weak form integrals have strong spatial variations, they can be included in the integrands $f_{i}$ (see Algorithm below) that are evaluated to construct the quadrature rule within the element. The following pseudo-code explains our quadrature construction scheme.

\section{Algorithm: Adaptive quadrature construction}

Input: The domain of integration $\Omega$; numf integrands $\left\{f_{i}\right\}$; prescribed error tolerance tol

Output: Adaptive quadrature over $\Omega$ that integrates $\left\{f_{i}\right\}_{i=1}^{\text {numf }}$ within the accuracy of tol for $i=1:$ numf do

$$
\begin{aligned}
& I_{1}=\text { integrate } f_{i} \text { over } \Omega \text { with } 5 \text {-point quadrature } \\
& I_{2}=\text { integrate } f_{i} \text { over } \Omega \text { with } 8 \text {-point quadrature } \\
& \text { if }\left|I_{2}-I_{1}\right|>\text { tol then set } P_{i}=1 \text {, otherwise set } P_{i}=0
\end{aligned}
$$

end do

if $\left\{P_{i}\right\}_{i=1}^{\text {numf }}=0$

return the 5-point quadrature as the rule over $\Omega$ 
else

partition $\Omega$ into eight uniform cells $\left\{\Omega_{j}\right\}_{j=1}^{8}$

for each cell $\Omega_{j}$, call the quadrature construction routine with the integrands

$\left\{f_{i} \text {, s.t. } P_{i}=1\right\}_{i=1}^{\text {numf }}$

put the eight obtained quadratures together: the adaptive quadrature over $\Omega$

end if

This adaptive integration scheme is similar to that of van Dooren and de Ridder [28], except that in Reference [28], the domain is subdivided in one direction (dimension) at a time, and all integrands are carried until the last iteration. Limiting subdivision to one dimension at each step can be useful if the integrands have a strong dependence on one of the spatial dimensions. Berntsen et al. [29] devise an analogous scheme with nonuniform subdivision, and improve the error estimate: the error is approximated by combining integrals over a cell and its children. Adaptive integration by octree subdivision is also used by Pieper [30], and the integration error is estimated as the difference between the integral over a cell and its children.

\subsection{Example}

Consider the functions $f_{1}(\mathbf{x})=10 \exp \left(-100 r_{1}^{2}\right)$ and $f_{2}(\mathbf{x})=100 \exp \left(-200 r_{2}^{2}\right)$ as the integrands for the quadrature construction, where $r_{1}$ is the distance from the point $\mathbf{x}$ to the origin, $r_{2}$ is the distance to $(.81, .62, .73)$, and the domain of integration is the unit cube. Figure 1a shows an adaptive quadrature over the unit cube with the above integrands and an error tolerance of $10^{-6}$ with 8875 integration points. A sequence of octree refinements of the domain is shown in Figure 1b-1e. The MATLAB ${ }^{\text {TM }}$ implementation of the adaptive quadrature is listed in Appendix I.

Copyright (C) 2011 John Wiley \& Sons, Ltd.

Int. J. Numer. Meth. Engng 2011; 00:1-28

Prepared using nmeauth.cls 


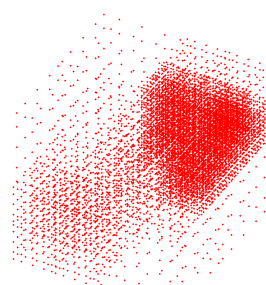

(a)

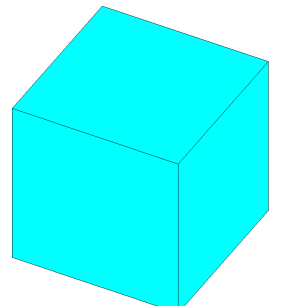

(b)

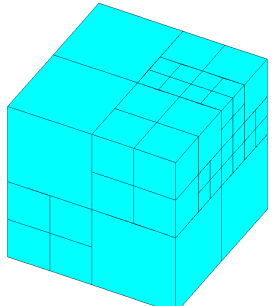

(c)

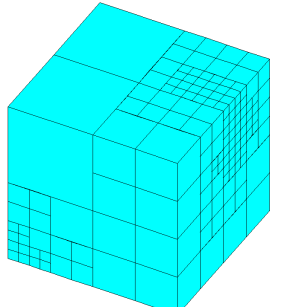

(d)

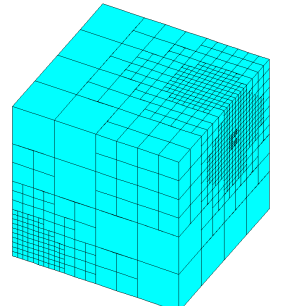

(e)

Figure 1. Adaptive quadrature over the unit cube. (a) quadrature points; and (b)-(e) the sequence of octree refinements.

\section{ERROR ANALYSIS}

In this section, the convergence properties of the adaptive integration algorithm are studied. Specifically, we are interested in cases where the integrand has a cusp, for example $f(\mathbf{x})=1-r$ or $f(\mathbf{x})=\exp (-\alpha r)$, which are $C^{0}$ functions with a derivative-discontinuity at the origin. However, this will not have an adverse effect on the efficiency of the adaptive integration scheme, and a high rate of convergence is realized. The following examples clarify the problem.

Consider the integration of the function $f(\mathbf{x})=1-r$, where $r$ is the distance of the point $\mathbf{x}$ to the origin. The integration domain is $[-1,1]^{n}$ for $n=1,2, \ldots, 6$. In Figure 2, the function $f$ is illustrated in one and two dimensions. Gauss quadratures are used to integrate $f$, and the position of the cusp is arbitrary. By contrast, one could consider the cusp as a hindrance to convergence, and partition the integration domain by placing the cusp at the boundary of the subdivisions. Figure 3 shows the convergence of the integration as the number of integration points is increased: a rate of convergence of $n+1$ is observed in $\mathbb{R}^{n}$, which can be explained by appealing to the Taylor expansion of the integrand. Since the integrand is continuous over a small cell $\Omega_{0}$ containing the cusp, the error of approximating it with a polynomial is $\mathcal{O}(h)$; hence the error in the integration is $\mathcal{O}(h) V_{\Omega_{0}} \sim \mathcal{O}\left(h^{n+1}\right)$, where $V_{\Omega_{0}} \sim \mathcal{O}\left(h^{n}\right)$ is 


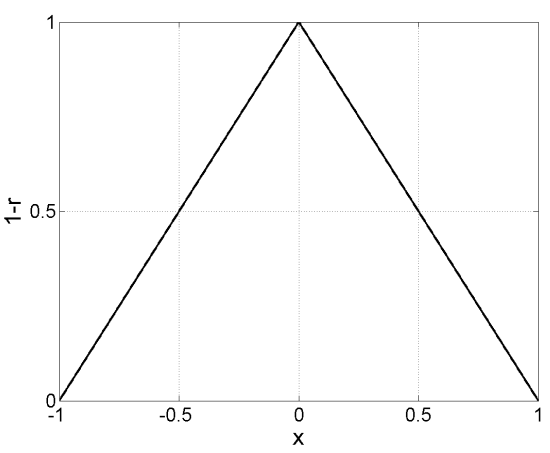

(a)

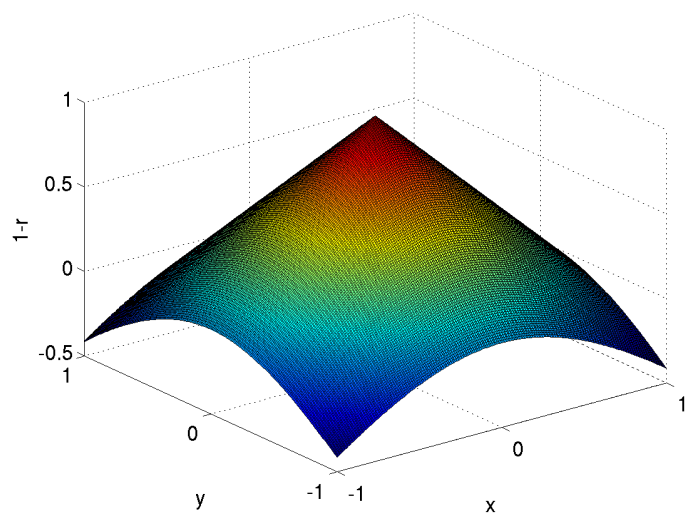

(b)

Figure 2. $f(\mathbf{x})=1-r$. (a) in one dimension; and (b) in two dimensions.

the volume of $\Omega_{0}$. This indicates that our quadrature construction scheme can attain higher convergence rates in higher dimensions (for $C^{0}$ functions), in contrast to the usual deficiency of numerical integration methods that suffer from the curse of dimensionality. We perform the same experiment with $f(\mathbf{x})=\exp (-20 r)$, which has a cusp at the origin. The function is plotted in Figure 4a in two dimensions. The convergence curves for the integration using tensor-product quadratures are shown in Figure 4b with respect to the minimum distance of the integration points to the cusp. As expected, a rate of convergence of $n+1$ is observed in $\mathbb{R}^{n}$. Numerical experiments also reveal that for smoother functions $C^{m}, m>0$, higher convergence rates are achieved, consistent with Darboux's Principle [31]. 


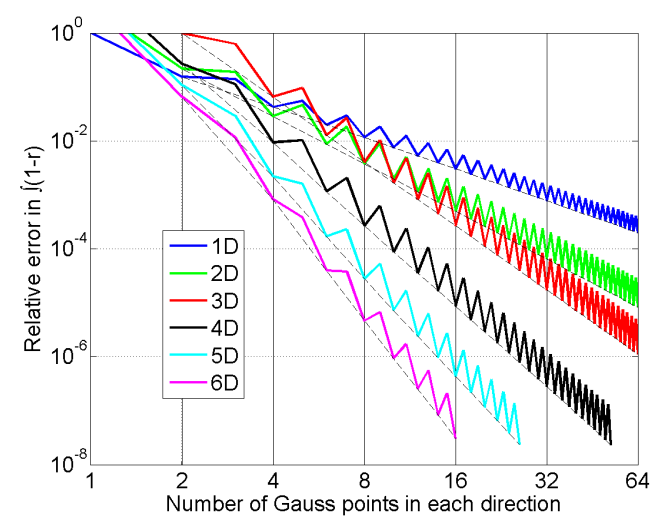

(a)

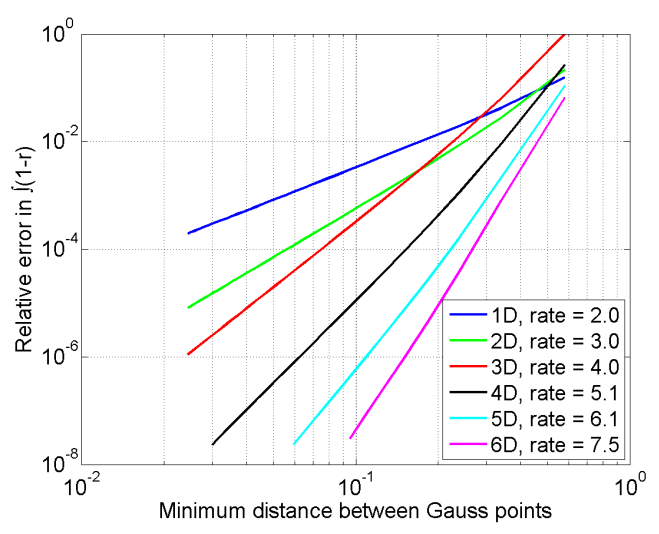

(b)

Figure 3. Convergence curves of the integration of $f(\mathbf{x})=1-r$. (a) with respect to the number of integration points in each direction; and (b) with respect to the minimum distance of the Gauss points to the cusp (only even number of integration points are shown).

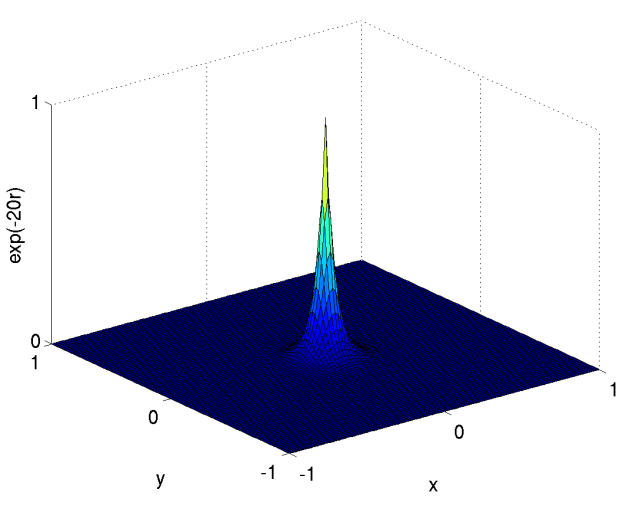

(a)

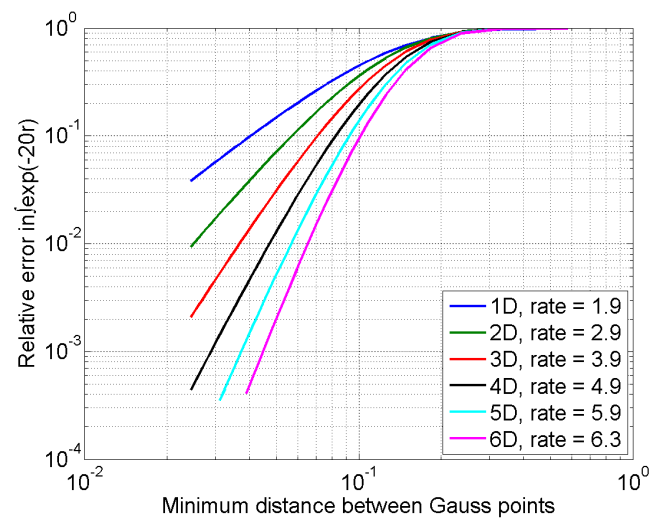

(b)

Figure 4. $f(\mathbf{x})=\exp (-20 r)$. (a) $f$ in two dimensions; and (b) convergence curves of the integration of $f(\mathbf{x})$ with respect to the minimum distance of the Gauss points to the cusp (only even number of integration points are shown). 


\section{NUMERICAL EXAMPLES}

\subsection{Integration of the Regularized Heaviside Function}

Solution fields with sharp spatial gradients arise in modeling physical phenomena such as shear band evolution, damage and cohesive process zones, and convection-dominated problems with shocks. Different classes of the regularized step function are adopted as the enrichment function for modeling sharp gradients, among which the following piecewise polynomial regularized Heaviside function is proposed by Patzák and Jirásek [1]:

$$
\psi(\phi, \varepsilon)= \begin{cases}0 & \text { if } \phi<-\varepsilon \\ \frac{1}{V_{\varepsilon}} \int_{-\varepsilon}^{\phi}\left(1-\frac{\xi^{2}}{\varepsilon^{2}}\right)^{4} d \xi & \text { if }|\phi| \leq \varepsilon \\ 1 & \text { if } \phi>\varepsilon,\end{cases}
$$

where $\phi(\mathbf{x})$ is the signed distance from the interface, and $\varepsilon$ is a parameter that determines the gradient of the enrichment function (half-width of the zone). The reference volume $V_{\varepsilon}$ is set to $256 \varepsilon / 315$ to enforce $C^{4}$ continuity. Integrating (1) gives [10]:

$$
\psi(\phi, \varepsilon)=\frac{1}{256 \varepsilon^{9}}\left(128 \varepsilon^{9}+315 \phi \varepsilon^{8}-420 \phi^{3} \varepsilon^{6}+378 \phi^{5} \varepsilon^{4}-180 \phi^{7} \varepsilon^{2}+35 \phi^{9}\right)
$$

in the region $|\phi| \leq \varepsilon$. It has been suggested that a single enrichment function may not be adequate to represent the complete range of sharp gradients present in a problem, e.g., the shock front in a convection-dominated problem, or the damage process zone in quasibrittle materials. For such problems, multiple enrichment functions, each having a separate parameter $\varepsilon$ should be used $[1,10]$. For the sake of illustration consider five functions with the parameter $\varepsilon / h=\{2.5,0.85,0.265,0.085,0.0225\}$ ( $h$ is the element size $)$, and integrate them on the unit square $(h=1)$. This set was produced by Abbas et al. [10] to minimize the pointwise error in modeling shocks. Other types of sharp-gradient enrichment functions, such as $\tanh (q \phi) \operatorname{can}$ 
also be used, where $q$ controls the severity of the gradient. The interface is a straight line shown in Figure 5a, and the enrichment function for $\varepsilon=0.085$ is depicted in Figure $5 f$. Four different integration strategies are examined: (1) tensor-product over the square, ignoring the interface; (2) and (3) integration over subtriangles using a coarse and a fine partitioning; and (4) adaptive quadrature. In all cases, the accuracy is improved by increasing the number of integration points (Figure 5g). The adaptive integration outperforms all other integration strategies. The experiment is repeated for a kinked interface and the results are shown in Figure 6 . Due to the shape of the interface, the integrand in this case is relatively more complicated, and the advantage of using the adaptive quadrature is emphasized. For an arbitrary curved discontinuity, different methods have been proposed, for example, integration by partitioning the element into triangles with curved edges [32,33], and octree-subdivision [34]. In Figure 7, the regularized Heaviside functions are integrated over a domain with a curved discontinuity. The interface is represented in closed form using a quadratic polynomial. The performance of the adaptive quadrature is compared with the tensor-product quadrature in Figure 7e. Adaptive quadratures require fewer integration points for the same accuracy.

\subsection{Enriched Finite Element in Quantum-Mechanical Calculations}

In Reference [14], an enriched finite element method was applied to the Coulomb problem in crystalline diamond, and tensor-product quadratures were used for the numerical integration. The number of integration points was increased until convergence in the solution was achieved. A higher-order quadrature was used over the elements containing the nuclei, since the enriched basis functions and the source term were strongly localized about the nuclei. A large number of integration points were required to obtain the desired accuracy and the optimal rate of 


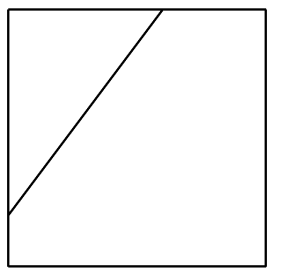

(a)

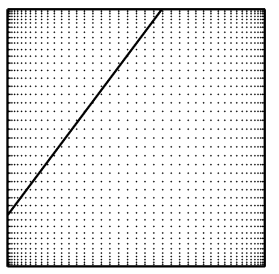

(b)

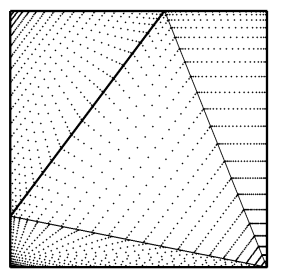

(c)

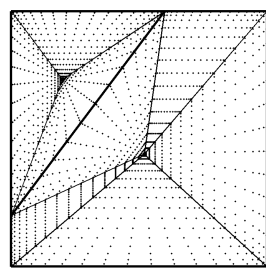

(d)

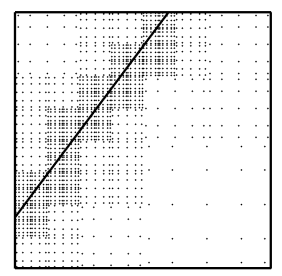

(e)

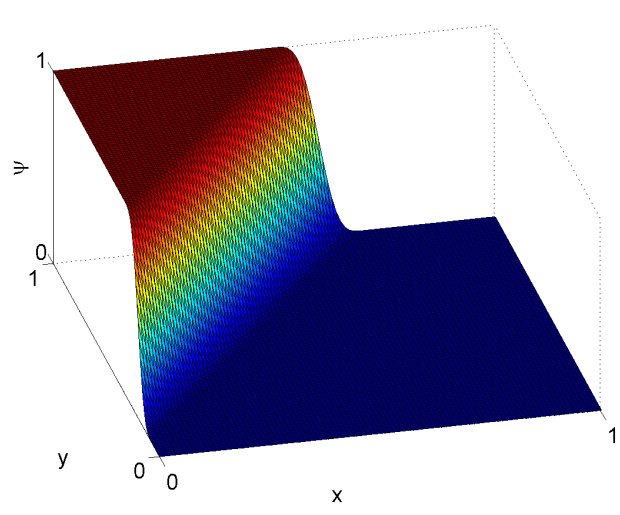

(f)

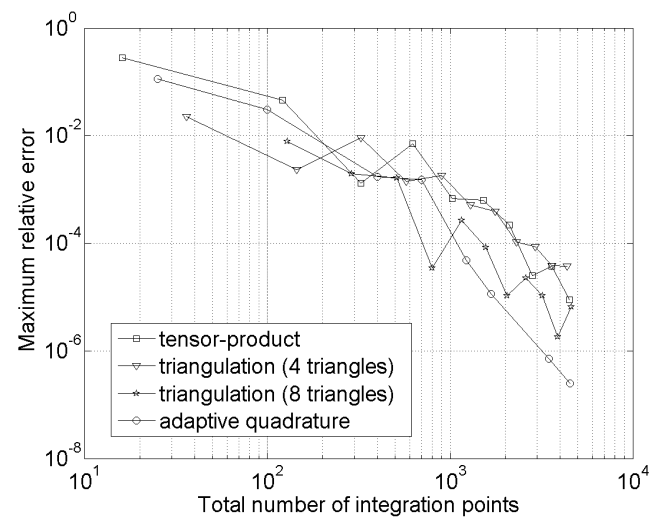

(g)

Figure 5. Numerical integration of the regularized Heaviside function with a straight interface. (a) domain of integration and interface; (b) tensor-product; (c) triangular quadratures (4 triangles); (d) triangular quadratures (8 triangles); (e) adaptive quadrature; (f) the regularized Heaviside function for $\varepsilon=0.085$; and $(\mathrm{g})$ maximum relative error in the integration of the five functions versus the number of integration points for different strategies.

convergence. Herein, we use the adaptive integration scheme introduced in Section 2 to setup the system matrices, and show that the optimal rate of convergence is realized at a relatively low computational cost. First, we present a brief description of the problem, and then the adaptive numerical integration algorithm is used to set up the finite element system matrices. For details on the formulation and solution technique, see References $[13,14]$. 


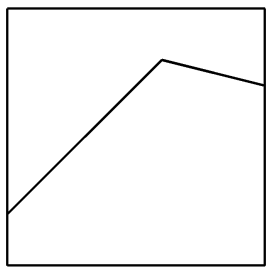

(a)

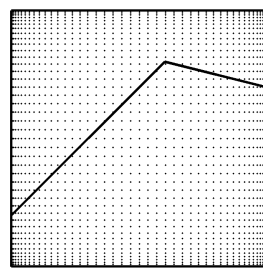

(b)

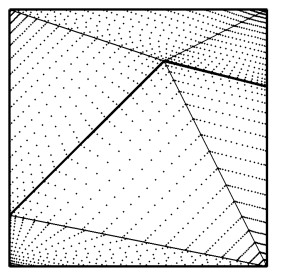

(c)

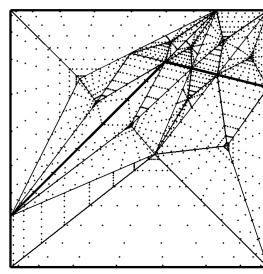

(d)

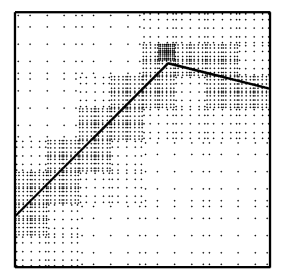

(e)

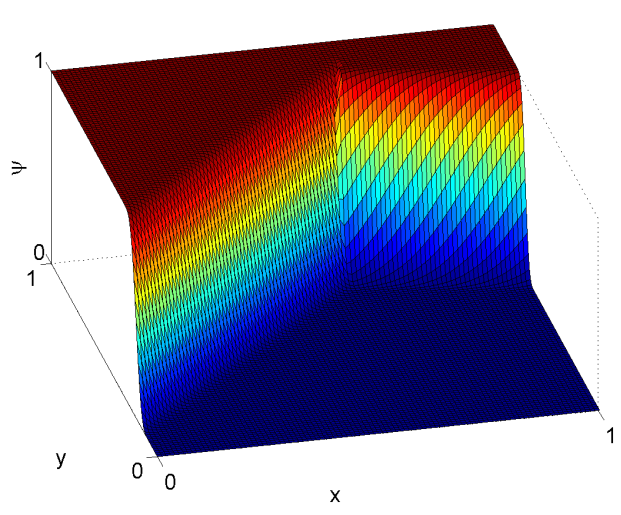

(f)

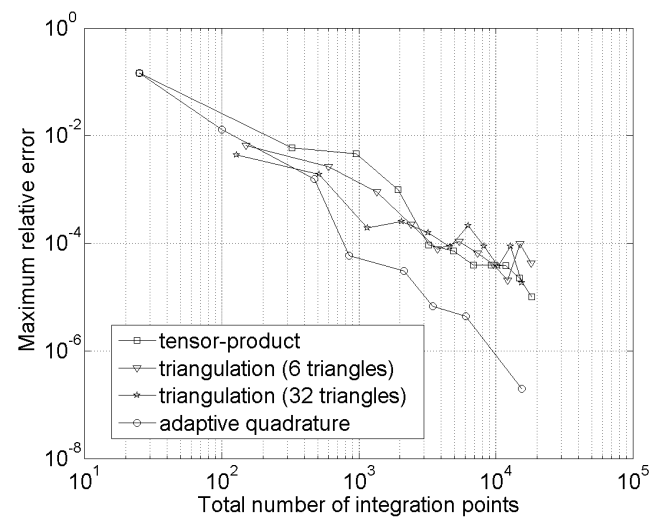

(g)

Figure 6. Numerical integration of the regularized Heaviside function with a kinked interface. (a) domain of integration and interface; (b) tensor-product; (c) triangular quadratures (6 triangles); (d) triangular quadratures (32 triangles); (e) adaptive quadrature; (f) the regularized Heaviside function for $\varepsilon=0.085$; and $(\mathrm{g})$ maximum relative error in the integration of the five functions versus the number of integration points for different strategies.

Consider the unit cell defined by the lattice vectors

$$
\begin{aligned}
& \mathbf{a}_{1}=\frac{a}{2}(0,1,1) \\
& \mathbf{a}_{2}=\frac{a}{2}(1,0,1) \\
& \mathbf{a}_{3}=\frac{a}{2}(1,1,0),
\end{aligned}
$$

where $a=6.75$ bohr, and carbon atoms are at $\boldsymbol{\tau}_{1}=(0,0,0)$ and $\boldsymbol{\tau}_{2}=(1 / 4,1 / 4,1 / 4)$ in lattice Copyright (C) 2011 John Wiley \& Sons, Ltd.

Int. J. Numer. Meth. Engng 2011; 00:1-28

Prepared using nmeauth.cls 


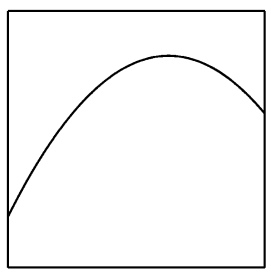

(a)

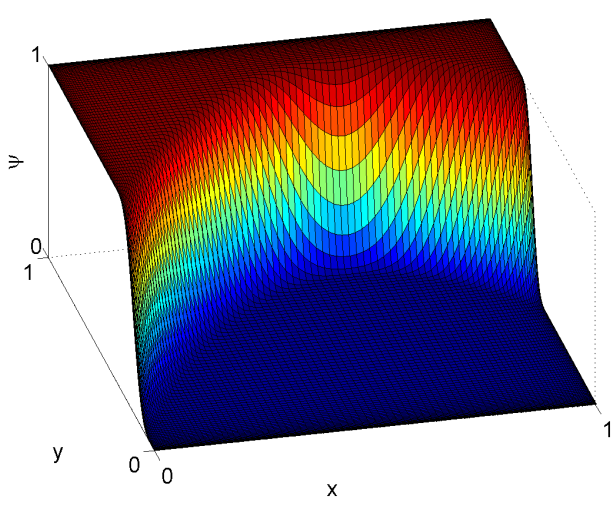

(d)

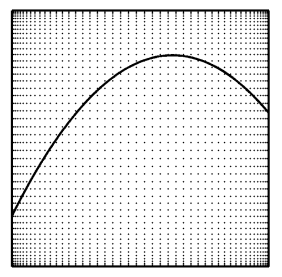

(b)

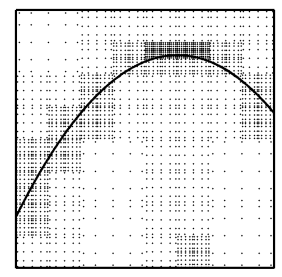

(c)

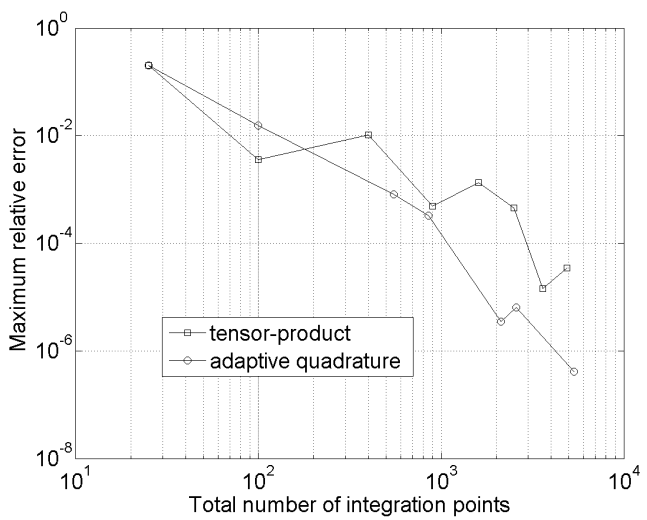

(e)

Figure 7. Numerical integration of the regularized Heaviside function with a curved interface. (a) domain of integration and interface; (b) tensor-product; (e) adaptive quadrature; (f) the regularized Heaviside function for $\varepsilon=0.085$; and $(\mathrm{g})$ maximum relative error in the integration of the five functions versus the number of integration points for different strategies.

coordinates. The total charge $\rho$ in the unit cell is written as

$$
\rho(\mathbf{x})=\rho^{+}(\mathbf{x})+\rho^{-}(\mathbf{x})=\rho^{+}(\mathbf{x})-\tilde{\rho}(\mathbf{x})+\rho^{-}(\mathbf{x})+\tilde{\rho}(\mathbf{x})=\tilde{\rho}^{+}(\mathbf{x})+\tilde{\rho}^{-}(\mathbf{x}),
$$

where $\rho^{+}(\mathbf{x})=\sum_{i} \rho_{i}(\mathbf{x})=\sum_{i} q_{i} \delta\left(\mathbf{x}-\boldsymbol{\tau}_{i}\right)$ is the total nuclear charge density in the unit cell, $\rho^{-}(\mathbf{x})$ is the electronic charge density, and $\tilde{\rho}^{+}(\mathbf{x})=\rho^{+}(\mathbf{x})-\tilde{\rho}(\mathbf{x})$ and $\tilde{\rho}^{-}(\mathbf{x})=\rho^{-}(\mathbf{x})+\tilde{\rho}(\mathbf{x})$ are the neutralized nuclear and electronic charge densities, respectively. The neutralizing charge $\tilde{\rho}(\mathbf{x})$ is introduced in (4) to circumvent the divergence of the potential $V^{+}(\mathbf{x})\left(V^{+} \sim 1 / r\right)$ at nuclear locations, so that $\tilde{V}^{+}$is extracted analytically, and $\tilde{V}^{-}$is solved in real space. The Copyright (C) 2011 John Wiley \& Sons, Ltd.

Int. J. Numer. Meth. Engng 2011; 00:1-28

Prepared using nmeauth.cls 
potential $\tilde{V}^{-}$associated with the neutralized electronic charge density is obtained from the solution of Poisson's equation:

$$
\nabla^{2} \tilde{V}^{-}(\mathbf{x})=-4 \pi \tilde{\rho}^{-}(\mathbf{x})
$$

subject to periodic boundary conditions, with continuous neutralized electronic charge density $\tilde{\rho}^{-}(\mathbf{x})$ as the source term. The EFE solution is written as

$$
\tilde{V}^{-}(\mathbf{x})=\sum_{i} \phi_{i}(\mathbf{x}) a_{i}+\sum_{\alpha} \psi_{\alpha}(\mathbf{x}) b_{\alpha} \equiv \sum_{k} \Phi_{k}(\mathbf{x}) c_{k},
$$

where $\alpha$ is summed over the atoms and $\left\{\Phi_{k}\right\}=\left\{\phi_{i}\right\} \cup\left\{\psi_{\alpha}\right\}$ is the combination of the classical and enriched basis functions that form the EFE basis. The enrichment functions $\psi_{\alpha}(\mathbf{x})$ are taken as sum of the potentials $\tilde{v}_{I}^{-}$-isolated atomic solutions corresponding to the neutralized electronic charge densities $\tilde{\rho}_{I}^{-}=\rho_{I}^{-}+\tilde{\rho}_{I}$ in the vicinity of each atom $I$. The enrichment function is written as

$$
\psi_{\alpha}(\mathbf{x})=\tilde{V}_{\alpha}^{-}(\mathbf{x})=\sum_{\mathbf{R}} \tilde{v}_{\alpha}^{-}(\mathbf{x}-\mathbf{R})
$$

where $\mathbf{R}$ denotes lattice translation vectors. The electronic charge densities and the enrichment functions are plotted in Figure 8.

On incorporating the trial and test functions of the form (5) into the weak form of the Poisson's equation, the discrete linear system of equations emerges. It is seen that the terms $\psi, \psi^{2}, \partial \psi / \partial r,(\partial \psi / \partial r)^{2}$, and $\psi \partial \psi / \partial r$ appear in the element stiffness matrix, and $\rho$ in the element force vector, where for simplicity $\psi$ and $\rho$ are used for the enrichment function, and the electronic charge density, respectively, and $\partial \psi / \partial r$ refers to the derivative with respect to the radial coordinate. These functions are normalized, with an absolute maximum value of unity, and plotted in Figure 9. The integrands have sharp gradients close to the atomic positions and produce cusps at the atomic sites. While it is possible to integrate these terms separately Copyright (C) 2011 John Wiley \& Sons, Ltd. Int. J. Numer. Meth. Engng 2011; 00:1-28

Prepared using nmeauth.cls 


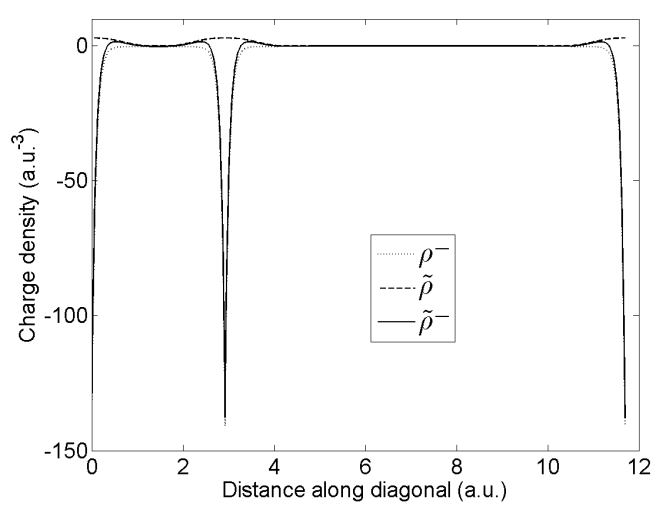

(a)

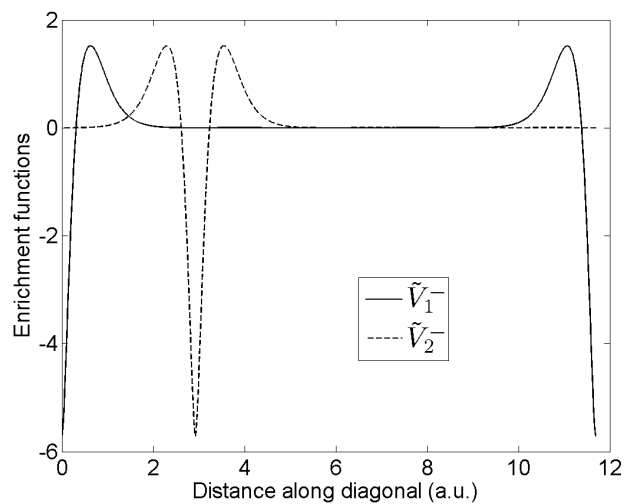

(b)

Figure 8. (a) Electronic charge densities; and (b) enrichment functions for the Coulomb problem in crystalline diamond [14].

(resulting in multiple ad hoc quadratures), it is desirable to have a single quadrature that is capable of efficiently evaluating the integrals altogether. The proposed numerical integration algorithm constructs a quadrature rule over each finite element that satisfies a given error tolerance for all the above integrands. Once the finite element mesh is generated, adaptive quadratures are constructed and saved for each element, which are used throughout the analysis and the postprocessing.

A sequence of refined meshes are used with 4, 8, 12, 16, 20, 24, and 32 cubic serendipity finite elements in each direction. Numerical integration is performed with tensor-product and adaptive quadratures. In each case, the accuracy of the quadratures is increased until convergence in the solution is observed. The number of integration points for each mesh and integration strategy is reported in Table I. Figure 10 shows the convergence curves for FE and EFE runs, with a rate of convergence of 4.39 and 5.96, respectively, for the last three data points. The results indicate that the integration scheme is sufficiently accurate to realize the optimal rate of convergence. The error tolerance (input of the quadrature construction Copyright (C) 2011 John Wiley \& Sons, Ltd. Int. J. Numer. Meth. Engng 2011; 00:1-28 


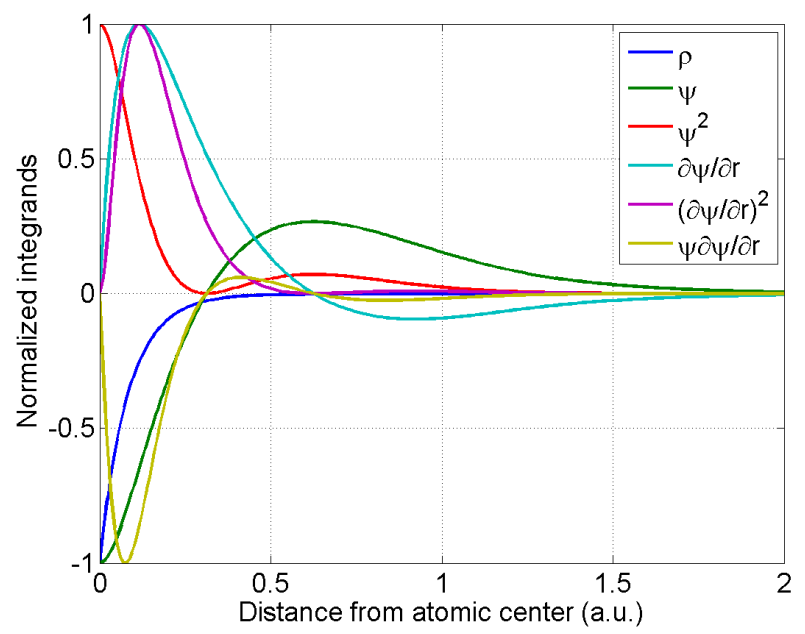

Figure 9. Normalized integrands in the element stiffness matrix and force vector of the Poisson problem.

algorithm) is the maximum allowable error that produces a stable result (i.e., stable with respect to further decrease in tolerance). For the pure FE runs, a 5-point Gauss quadrature rule is used in each direction. Adaptive integration proves to be superior with respect to the tensorproduct quadrature. The improvement is emphasized for finer meshes where higher accuracies are required: the integration demand of the EFE solution is only marginally higher than the pure FE solution (of much lower accuracy) on the same mesh. In Figure 11, a comparison is made between the behavior of tensor-product and adaptive quadratures for the EFE solution of the Coulomb problem in crystalline diamond. The quadrature error in the Coulomb energy is plotted with respect to the number of integration points for finite element meshes with 4 and 16 elements in each direction. The accuracy of the quadrature is increased until the desired convergence with respect to the quadrature is attained. The adaptive quadrature requires fewer integration points to achieve the same accuracy. Standard Gauss quadrature shows a smoother convergence curve, which can be attributed to the uniform overall increase in the Copyright (C) 2011 John Wiley \& Sons, Ltd. Int. J. Numer. Meth. Engng 2011; 00:1-28 Prepared using nmeauth.cls 
accuracy of the quadrature throughout the domain. The efficiency of the adaptive quadrature is more noticeable in case of the $16 \times 16 \times 16$ mesh.

Note that in all the meshes used earlier, the atoms are located at the vertices of the finite elements - the atom inside the unit cell is at a quarter of the diagonal from the corner, and the number of elements is a multiple of four - and there is no atom inside any finite element. For more general lattice systems, non-uniformly refined meshes may be required in order to ensure that all atoms are located at the vertices of elements. This is not desirable due to the increase in the total number of elements and the associated computational costs. Furthermore, with the application of the EFE, one would like to resolve the local features by adding enrichment functions to the approximation, and not by refining the finite element mesh. In the following, we use meshes with $1,2,3,5,6$, and 7 elements in each direction. In all these cases, the atom in the unit cell lies inside an element. The number of integration points of the tensorproduct and adaptive quadratures are compared in Table II. The increase in the number of integration points for the tensor-product quadrature is significant, whereas the adaptive integration provides accurate results with a moderate number of integration points.

\section{CONCLUDING REMARKS}

An adaptive integration scheme was presented that can be used for functions with sharp gradients and cusps. The algorithm uses tensor-product quadratures with 5 and 8 integration points in each direction to estimate the local error, and divides the domain uniformly, independent of the location of the cusp or sharp gradient, until the target tolerance is met. The error analysis in the integration of a function with a cusp (derivative-discontinuity at a point) showed that the rate of convergence is improved in higher dimensions. The adaptive 
Table I. Comparison of the number of integration points for tensor-product and adaptive quadratures.

\begin{tabular}{lllll}
\hline \multirow{2}{*}{ Mesh } & Error tolerance & Number of integration points & \\
\cline { 3 - 5 } & & Pure FE & Tensor-product & Adaptive \\
\hline 4 & $2.2 \times 10^{-3}$ & 8000 & 169000 & 78000 \\
8 & $1.7 \times 10^{-4}$ & 64000 & 624000 & 162000 \\
12 & $2.2 \times 10^{-5}$ & 216000 & 1840000 & 349000 \\
16 & $4.3 \times 10^{-6}$ & 512000 & 14020000 & 981000 \\
20 & $1.1 \times 10^{-6}$ & 1000000 & 27196000 & 1549500 \\
32 & $4.7 \times 10^{-8}$ & 4096000 & 46852000 & 3073750 \\
\hline
\end{tabular}

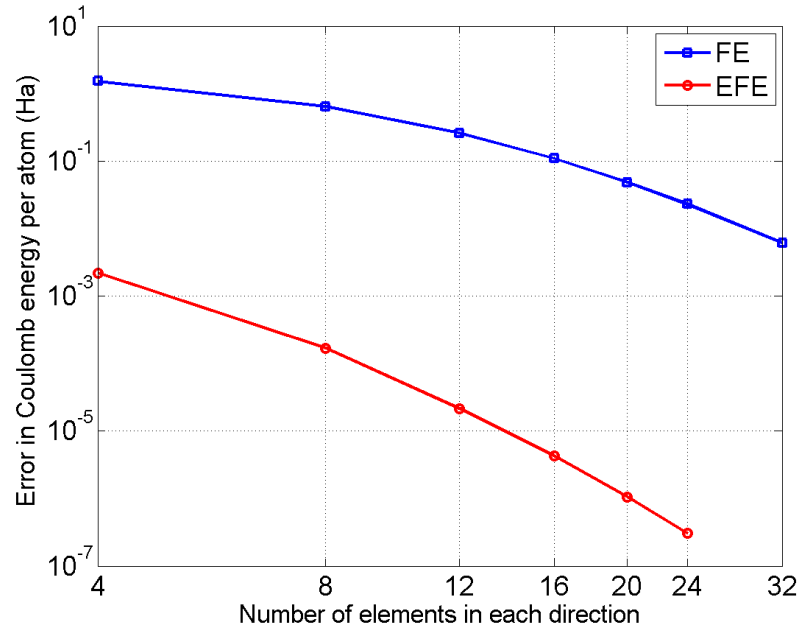

Figure 10. Error in Coulomb energy per atom. $32 \times 32 \times 32$ mesh is used as the reference solution. 


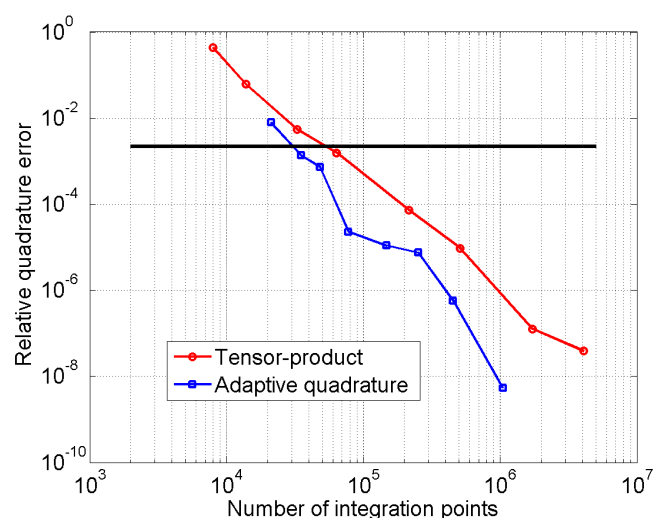

(a)

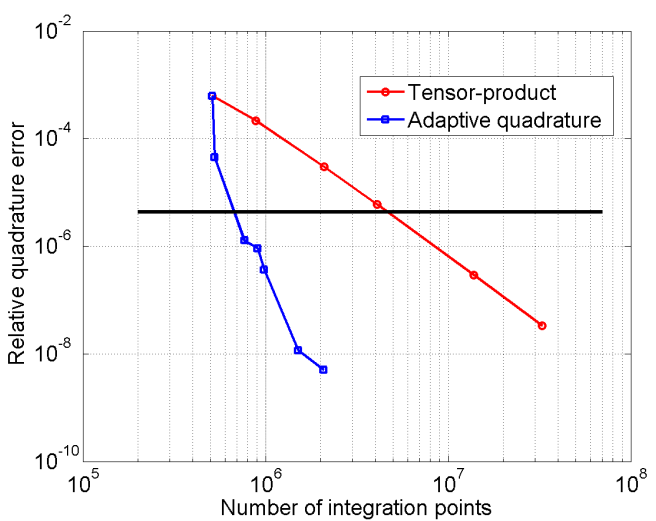

(b)

Figure 11. Comparison of tensor-product and adaptive quadratures for the crystalline diamond problem. The thick line shows the EFE solution error. (a) $4 \times 4 \times 4$ mesh; and (b) $16 \times 16 \times 16$ mesh.

Table II. Comparison of the number of integration points for tensor-product and adaptive quadratures: one of the atoms is inside an element.

\begin{tabular}{lllll}
\hline \multirow{2}{*}{ Mesh } & Error tolerance & Number of integration points & \\
\cline { 3 - 5 } & & Pure FE & Tensor-product & Adaptive \\
\hline 1 & $9.8 \times 10^{-3}$ & 125 & 1000000 & 48250 \\
2 & $6.8 \times 10^{-3}$ & 1000 & 4096000 & 48250 \\
3 & $3.3 \times 10^{-3}$ & 3375 & 1880000 & 55875 \\
5 & $1.1 \times 10^{-3}$ & 15625 & 8000000 & 165250 \\
6 & $4.9 \times 10^{-4}$ & 27000 & 27000000 & 188875 \\
7 & $3.0 \times 10^{-4}$ & 42875 & 21952000 & 183750 \\
\hline
\end{tabular}


integration algorithm was successfully used for the integration of a set of regularized Heaviside functions, and proved to be more efficient than integration by partitioning as well as tensorproduct quadratures. The method was also applied to the enriched finite element solution of the all-electron Coulomb potential and energy of crystalline diamond (Poisson's equation). The enrichment functions and source term were strongly localized about the atomic positions. The adaptive integration scheme proved to be very efficient, and recovered the optimal rate of convergence with only a moderate increase in the number of integration points with respect to the classical finite element method (of much lower accuracy) on the same mesh; while reducing the integration points required in the EFE solution by an order of magnitude or more. The adaptive integration scheme is simple, robust, and directly applicable to any generalized finite element method employing enrichments with sharp local variations or cusps in $n$-dimensional parallelepiped elements.

\section{ACKNOWLEDGEMENTS}

This work was performed in part under the auspices of the U.S. Department of Energy by Lawrence Livermore National Laboratory under Contract DE-AC52-07NA27344. S. E. Mousavi and N. Sukumar are grateful for the research support of the National Science Foundation through contract grant DMS-0811025 to the University of California at Davis; additional financial support from the UC Lab Fees Research Program is also acknowledged.

\section{REFERENCES}

1. B. Patzák and M. Jirásek. Process zone resolution by extended finite elements. Engineering Fracture Mechanics, 70:957-977, 2003.

Copyright (C) 2011 John Wiley \& Sons, Ltd.

Int. J. Numer. Meth. Engng 2011; 00:1-28

Prepared using nmeauth.cls 
2. E. Benvenuti, A. Tralli, and G. Ventura. A regularized XFEM model for the transition from continuous to discontinuous displacements. International Journal for Numerical Methods in Engineering, 74:911-944, 2008.

3. E. Benvenuti. A regularized XFEM framework for embedded cohesive interfaces. Computer Methods in Applied Mechanics and Engineering, 197:4367-4378, 2008.

4. P. M. A. Areias and T. Belytschko. Two-scale shear band evolution by local partition of unity. International Journal for Numerical Methods in Engineering, 66:878-910, 2006.

5. P. M. A. Areias and T. Belytschko. Two-scale method for shear bands: thermal effects and variable bandwidth. International Journal for Numerical Methods in Engineering, 72:658-696, 2007.

6. K. K. Tamma and K. C. Saw. Hierarchical p-version finite elements and adaptive a posteriori computational formulations for two-dimensional thermal analysis. Computers and Structures, 32(5):1989, 1183-1194.

7. R. Merle and J. Dolbow. Solving thermal and phase change problems with the eXtended finite element method. Computational Mechanics, 28(5):339-350, 2002.

8. P. O'Hara, C. A. Duarte, and T. Eason. Generalized finite element analysis of three-dimensional heat transfer problems exhibiting sharp thermal gradients. Computer Methods in Applied Mechanics and Engineering, 198:1857-1871, 2009.

9. A. N. Brooks and T. J. R. Hughes. Streamline upwind/Petrov-Galerkin formulations for convection dominated flows with particular emphasis on the incompressible Navier-Stokes equations. Computer Methods in Applied Mechanics and Engineering, 32(1-3):199-259, 1982.

10. S. Abbas, A. Alizada, and T. P. Fries. The XFEM for high-gradient solutions in convection-dominated problems. International Journal for Numerical Methods in Engineering, 82:1044-1072, 2010.

11. I. Kalashnikova, R. Tezaur, and C. Farhat. A discontinuous enrichment method for variable-coefficient advection-diffusion at high Péclet number. International Journal for Numerical Methods in Engineering, 87:309-335, 2011.

12. M. Challacombe. Linear scaling computation of the Fock matrix. V. Hierarchical cubature for numerical integration of the exchange-correlation matrix. Journal of Chemical Physics, 113(22):10037-10043, 2000.

13. N. Sukumar and J. E. Pask. Classical and enriched finite element formulations for Bloch-periodic boundary conditions. International Journal for Numerical Methods in Engineering, 77(8):1121-1138, 2009.

14. J. E. Pask, N. Sukumar, and S. E. Mousavi. Linear scaling solution of the all-electron Coulomb problem in solids. International Journal for Multiscale Computational Engineering, 2011, in press. Available at 
arXiv: 1004.1765

15. J. M. Melenk and I. Babuška. The partition of unity finite element method: Basic theory and applications. Computer Methods in Applied Mechanics and Engineering, 139:289-314, 1996.

16. J. Berntsen, R. Cools, and T. O. Espelid. Algorithm 720 - An algorithm for adaptive cubature over a collection of 3-dimensional simplices. ACM Transactions on Mathematical Software, 19(3):320-332, 1993.

17. W. Gander and W. Gautschi. Adaptive quadrature-revisited. BIT Numerical Mathematics, 40(1):84$101,2000$.

18. J. Berntsen and T. O. Espelid. Algorithm-706 - DCUTRI: An algorithm for adaptive cubature over a collection of triangles. ACM Transactions on Mathematical Software, 18(3):329-342, 1992.

19. A. Genz and R. Cools. An adaptive numerical cubature algorithm for simplices. ACM Transactions on Mathematical Software, 29(3):297-308, 2003.

20. J. N. Lyness. Symmetric integration rules for hypercubes III. Construction of integration rules using null rules. Mathematics of Computation, 19(92):625-637, 1965.

21. J. Berntsen and T. O. Espelid. Error estimation in automatic quadrature routines. ACM Transactions on Mathematical Software, 17(2):233-252, 1991.

22. A.-K. Tornberg. Multi-dimensional quadrature of singular and discontinuous functions. BIT Numerical Mathematics, 42(3):644-669, 2002.

23. H. Oh, J. G. Kim, and J. W. Jeong. The smooth piecewise polynomial particle shape functions corresponding to patch-wise non-uniformly spaced particles for meshfree particle methods. Computational Mechanics, 40:569-594, 2007.

24. G. Ventura. On the elimination of quadrature subcells for discontinuous functions in the eXtended FiniteElement Method. International Journal for Numerical Methods in Engineering, 66:761-795, 2006.

25. H. H. H. Homeier and E. O. Steinborn. Numerical integration of functions with a sharp peak at or near one boundary using Möbius transformation. Journal of Computational Physics, 87:61-72, 1990.

26. H. H. H. Homeier and E. O. Steinborn. On the evaluation of overlap integrals with exponential-type basis functions. International Journal of Quantum Chemistry, 42:761-778, 1992.

27. R. López and G. Ramírez. Calculation of two-center exchange integrals with STOs using Möbius transformations. International Journal of Quantum Chemistry, 49:11-19, 1994.

28. P. van Dooren and L. de Ridder. An adaptive algorithm for numerical integration over an $n$-dimensional cube. Journal of Computational and Applied Mathematics, 2(3):207-217, 1976.

29. J. Berntsen, T. O. Espelid, and A. Genz. An adaptive algorithm for the approximate calculation of Copyright (C) 2011 John Wiley \& Sons, Ltd. Int. J. Numer. Meth. Engng 2011; 00:1-28

Prepared using nmeauth.cls 
multiple integrals. ACM Transactions on Mathematical Software, 17(4):437-451, 1991.

30. W. M. Pieper. Recursive Gauss integration. Communications in Numerical Methods in Engineering, 15(2):77-90, 1999.

31. J. P. Boyd. Chebyshev and Fourier spectral methods. Dover Publications, New York, NY, second edition, 2001.

32. A. Legay, H. W. Wang, and T. Belytschko. Strong and weak arbitrary discontinuities in spectral finite elements. International Journal for Numerical Methods in Engineering, 64:991-1008, 2005.

33. K. W. Cheng and T. P. Fries. Higher-order XFEM for curved strong and weak discontinuities. International Journal for Numerical Methods in Engineering, 82:564-590, 2010.

34. K. Dréau, N. Chevaugeon, and N. Moës. Studied X-FEM enrichment to handle material interfaces with higher order finite element. Computer Methods in Applied Mechanics and Engineering, 199:1922-1936, 2010 .

\section{APPENDIX}

\section{MATLAB Code for the Adaptive Integration Scheme}

The following routine is the implementation of our adaptive integration scheme and produces a quadrature over an $n$-dimensional hyperparallelepiped for a given set of functions and prescribed error tolerance. First, a description of the input and output parameters is given.

- $\mathrm{fn}: 1 \times$ numf, set of integrands, defined as an array of structures with the member $\mathrm{h}$, which is a function handle. For example, in case of two integrands, we have: $\mathrm{fn}(1) \cdot \mathrm{h}=$ Qintegrand 1 ; and $\mathrm{fn}(2) . \mathrm{h}=$ Qintegrand 2 ; where integrand $1 . \mathrm{m}$ and integrand2.m are MATLAB functions defined as $\mathbb{R}^{n} \rightarrow \mathbb{R}$.

- $\mathrm{d}$ : $(n+1) \times n$, domain of integration, a hyperparallelepiped in $\mathbb{R}^{n}$, defined using one point as its base and only $n$ subsequent vertices of $d$. For example, while $d(2,:)$ is a vertex of $d$, the vector $d(2,:)-d(1,:)$ is a lattice vector of $d$. 
- nsp: $1 \times 2$, number of integration points in each direction to evaluate the integral over the partitions. nsp(1) is used to evaluate the local integrals, and nsp(2) is used to evaluate the local integration error.

- tol: $1 \times 1$, absolute value of the integration error.

- $\mathrm{X}$ : $n \times n u m x$, quadrature points, each point is a column vector.

- W: $n u m x \times 1$, quadrature weights.

For example, the functions used in Section 2.1 are defined in the following m-files:

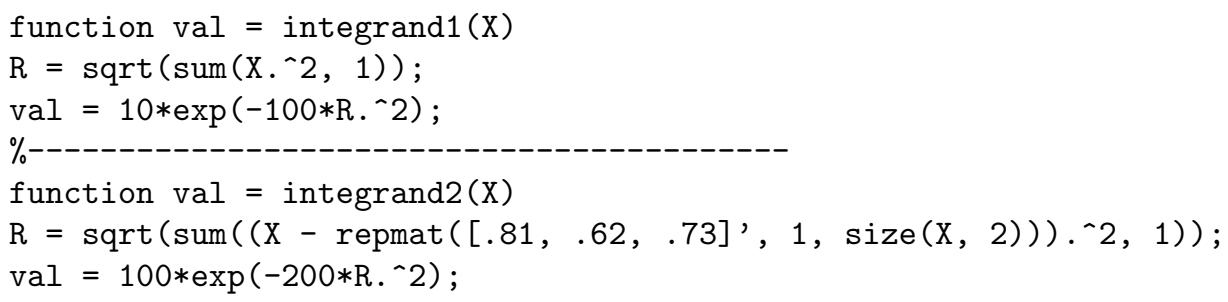

The domain of integration (unit cube) is defined using its base and three of its vertices:

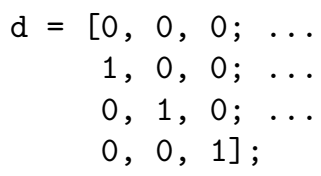

With the above setting, the adaptive quadrature function can be called (see Figure 1):

$[X, W]=$ ndimensional_adaptive_integration $(\mathrm{fn}, \mathrm{d},[5,8], 1 \mathrm{e}-6)$;

The MATLAB code for the quadrature construction follows.

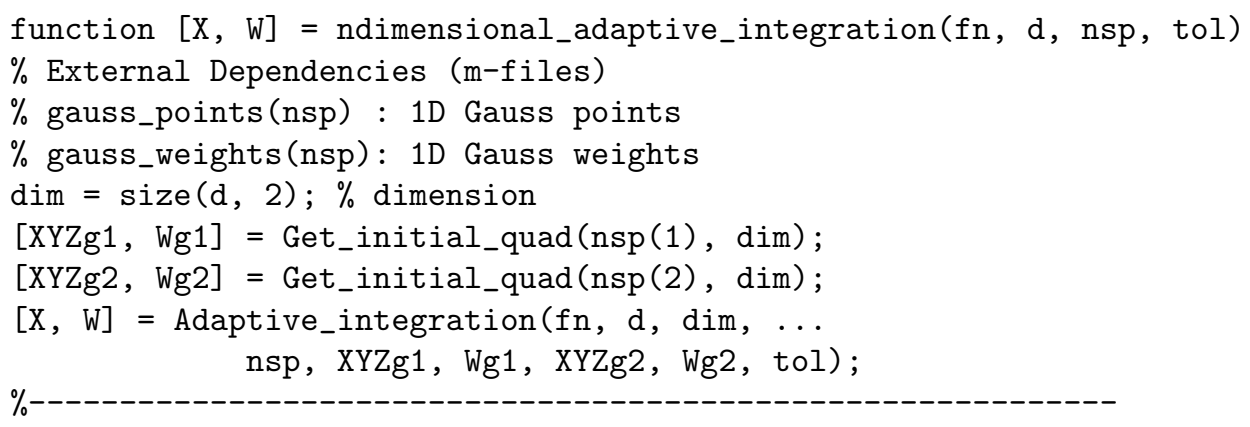




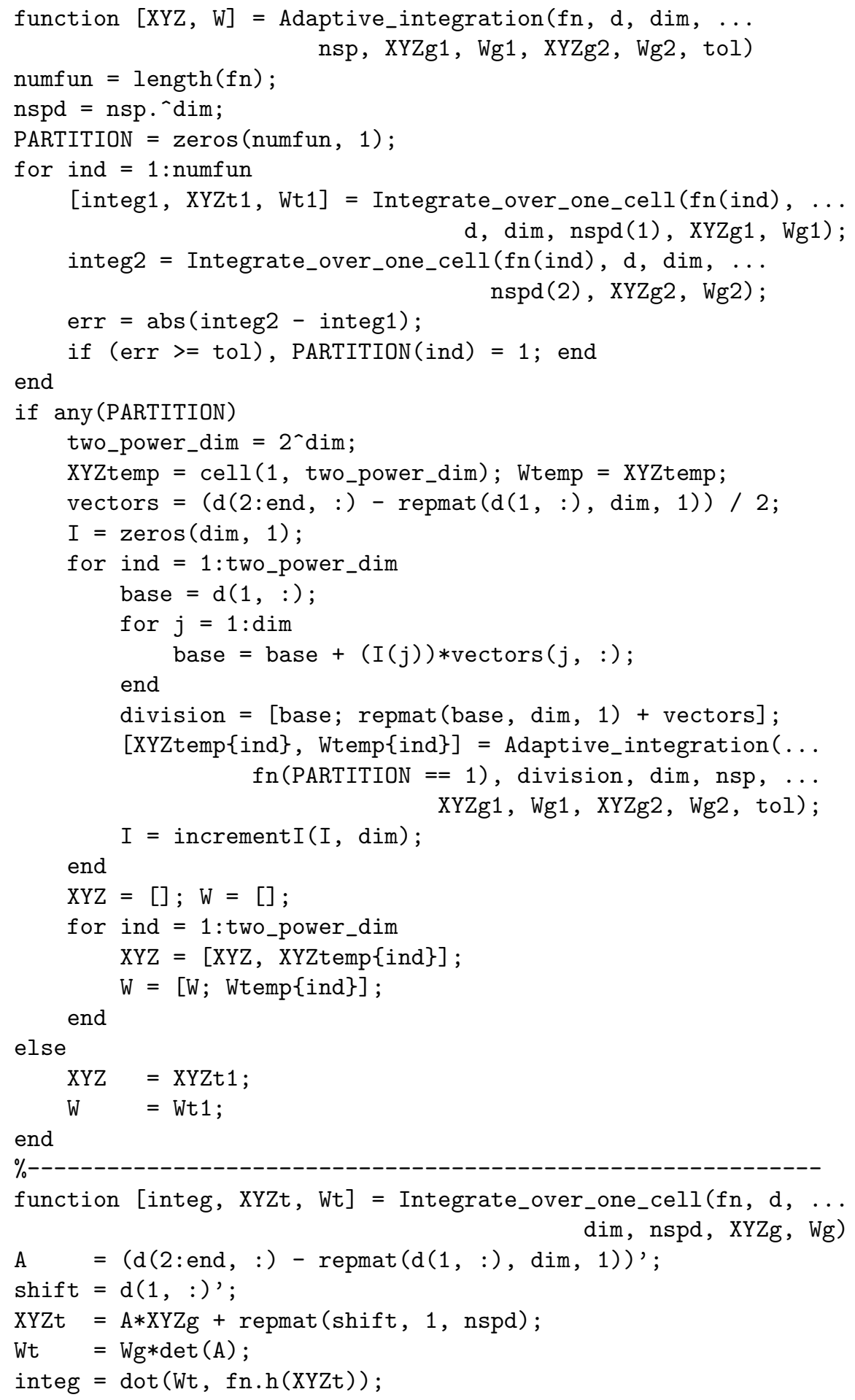




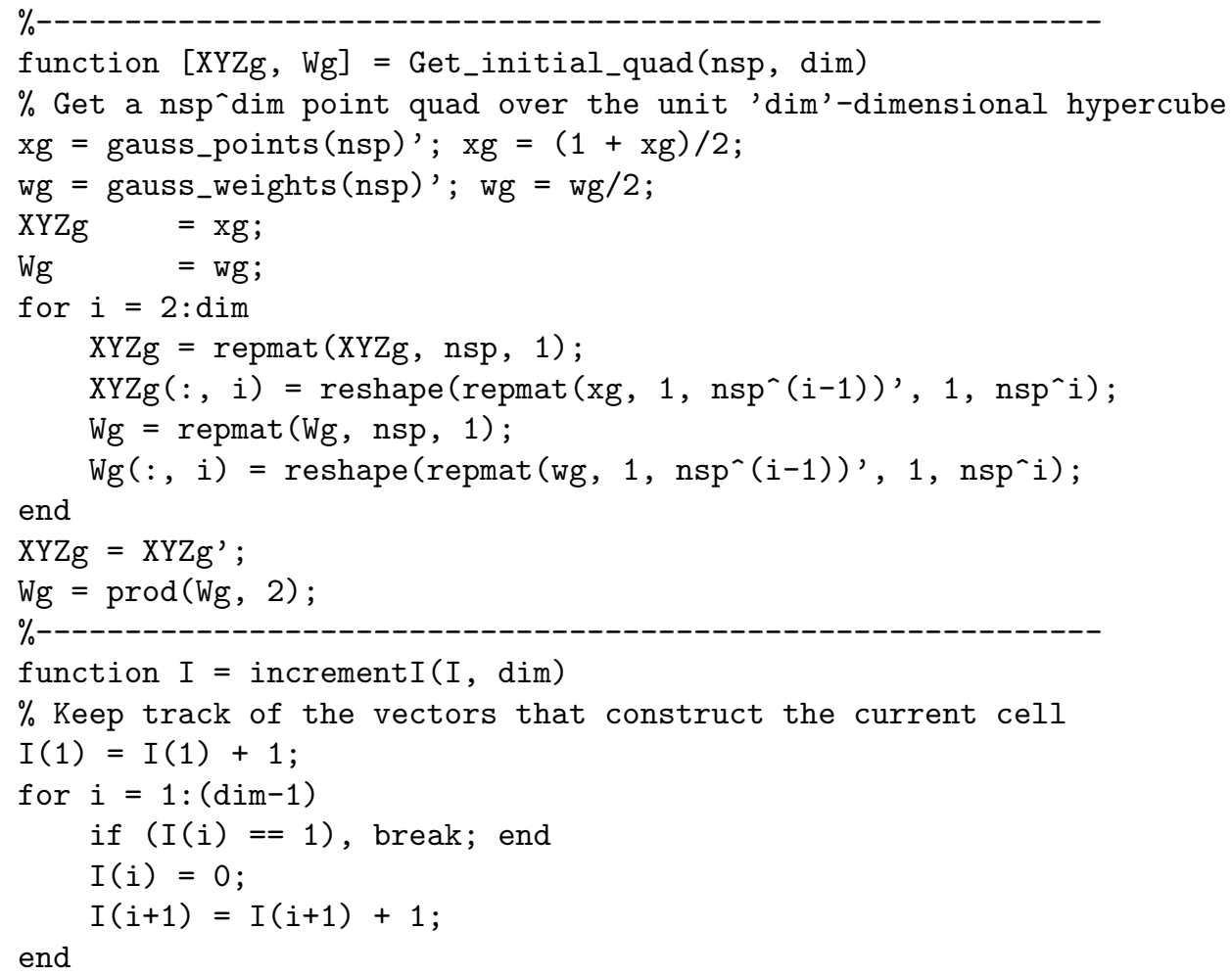

